\title{
Low lifetime stress exposure is associated with reduced stimulus-response memory
}

\author{
Elizabeth V. Goldfarb, ${ }^{1}$ Grant S. Shields, ${ }^{2}$ Nathaniel D. Daw, ${ }^{3,4}$ George M. Slavich, ${ }^{5,6}$ \\ and Elizabeth A. Phelps ${ }^{1,7,8}$
}

${ }^{1}$ Department of Psychology, New York University, New York, New York 10003, USA; ${ }^{2}$ Department of Psychology, University of California, Davis, Davis, California 95616, USA; ${ }^{3}$ Princeton Neuroscience Institute, Princeton University, Princeton, New Jersey 08544 , USA; ${ }^{4}$ Department of Psychology, Princeton University, Princeton, New Jersey 08544, USA; ${ }^{5}$ Cousins Center for

Psychoneuroimmunology, University of California, Los Angeles, Los Angeles, California 90095, USA; ${ }^{6}$ Department of Psychiatry and Biobehavioral Sciences, University of California, Los Angeles, Los Angeles, California 90095, USA; ${ }^{7}$ Center for Neural Science, New York University, New York, New York 10003, USA; ${ }^{8}$ Nathan Kline Institute, Orangeburg, New York 10962, USA

\begin{abstract}
Exposure to stress throughout life can cumulatively influence later health, even among young adults. The negative effects of high cumulative stress exposure are well-known, and a shift from episodic to stimulus-response memory has been proposed to underlie forms of psychopathology that are related to high lifetime stress. At the other extreme, effects of very low stress exposure are mixed, with some studies reporting that low stress leads to better outcomes, while others demonstrate that low stress is associated with diminished resilience and negative outcomes. However, the influence of very low lifetime stress exposure on episodic and stimulus-response memory is unknown. Here we use a lifetime stress assessment system (STRAIN) to assess cumulative lifetime stress exposure and measure memory performance in young adults reporting very low and moderate levels of lifetime stress exposure. Relative to moderate levels of stress, very low levels of lifetime stress were associated with reduced use and retention ( $24 \mathrm{~h}$ later) of stimulus-response (SR) associations, and a higher likelihood of using context memory. Further, computational modeling revealed that participants with low levels of stress exhibited worse expression of memory for SR associations than those with moderate stress. These results demonstrate that very low levels of stress exposure can have negative effects on cognition.
\end{abstract}

[Supplemental material is available for this article.]

Stressors occurring over the life course exert a cumulative impact on human health (McEwen 1998; Lupien et al. 2009). Even among preclinical samples of young adults, higher levels of lifetime stress exposure have been associated with adverse health outcomes (Toussaint et al. 2016), changes in reactivity to acute stress (Elzinga et al. 2008; Lovallo et al. 2012; Seery et al. 2013), and increased risk for psychopathology (Lloyd and Turner 2008). Stress-related psychopathologies may be driven in part by stress-induced memory biases. Specifically, an imbalance between flexible, rapidly acquired contextual associations (which depend on the hippocampus; Bunsey and Eichenbaum 1996; Burgess et al. 2002) and rigid, slowly acquired stimulus-response associations (which depend on the striatum; Graybiel 1998; Bayley et al. 2005; Yin and Knowlton 2006) has been theorized to contribute to disorders like addiction and post-traumatic stress disorder (PTSD; McDonald et al. 2004; Goodman and Packard 2016). For example, preferential use of stimulus-response memory could facilitate habitual drug-seeking behavior. However, the effects of lifetime stress exposure-especially very low levels of lifetime stress-on the relative use of contextual and stimulus-response memory are not yet known.

Prior research has suggested that the relative engagement of these memory systems may be sensitive to lifetime stress exposure. Across species, high levels of recent acute stress (Kim et al. 2001; Packard and Wingard 2004; Schwabe et al. 2007; 2010;

\section{Corresponding author: liz.phelps@nyu.edu}

Article is online at http://www.learnmem.org/cgi/doi/10.1101/lm.045179. 117.
Elliott and Packard 2008; Schwabe and Wolf 2012; Leong and Packard 2014) and chronic stress (Schwabe et al. 2008; Ferragud et al. 2010; Taylor et al. 2014) have been shown to facilitate preferential engagement of striatal rather than hippocampal memory. Importantly, remote stressors are also associated with a bias toward striatal memory (Schwabe et al. 2012; Patterson et al. 2013), indicating that stressors experienced throughout life may modulate memory systems. Further, periods of chronic stress can lead to lasting morphological changes that would facilitate this shift between memory systems. Chronic stress has frequently been shown to impair the structure and function of the hippocampus (McEwen 1999; Lupien et al. 2007) and has recently been shown to enhance the structure and function of the striatum (Dias-Ferreira et al. 2009; Taylor et al. 2014). Thus, just as chronic stress early in life can influence working memory in adulthood (Evans and Schamberg 2009), it may also modulate long-term memory systems.

Although these results lead to strong predictions regarding the effects of high levels of lifetime stress exposure, the possible effects of very low stress are less clear. Understanding the influence of low levels of stress on cognition is critical, as low lifetime stress exposure can lead to negative health outcomes and

\footnotetext{
(C) 2017 Goldfarb et al. This article is distributed exclusively by Cold Spring Harbor Laboratory Press for the first 12 months after the full-issue publication date (see http://learnmem.cshlp.org/site/misc/terms.xhtml). After 12 months, it is available under a Creative Commons License (AttributionNonCommercial 4.0 International), as described at http://creativecommons. org/licenses/by-nc/4.0/.
} 
diminished resilience (Dienstbier 1989; Seery et al. 2010, 2013; Liu 2015), even in young adult populations (Seery et al. 2013). This research has demonstrated that the effects of low and high lifetime stress exposure can be similarly negative. Although there have not been studies examining the effects of low lifetime stress on hippocampal memory, there is evidence that low (and high) physiological arousal responses can impair this form of memory (McCullough et al. 2015). These results suggest that low levels of lifetime stress exposure could be associated with impaired hippocampal memory. A separate literature on the effects of lifetime stress leads to different predictions about memory. These studies report that low and high lifetime stress exposure have opposite effects, with lower levels of stress associated with better outcomes (Lloyd and Turner 2008; Toussaint et al. 2016). For example, higher lifetime stress exposure correlates with increasing addiction risk (Lloyd and Turner 2008). Greater addiction risk is itself associated with stronger striatal memory (McDonald et al. 2004). Thus, if higher lifetime stress exposure is associated with stronger striatal memory, we may expect lower lifetime stress to be associated with weaker striatal memory.

In this study, we investigate the influence of low levels of lifetime stress exposure on contextual and stimulus-response memory. We measured cumulative lifetime stress experiences in a large cohort of undergraduate students at a 4-yr university, a sample previously shown to have relatively low stress exposure (Breslau et al. 1991; Anders et al. 2012). To compare participants with very low stress exposure to those with moderate stress exposure, we recruited individuals from the top and bottom of this distribution. We assessed participants' lifetime stress exposure using a comprehensive online interviewing system, and their memory using a recently developed task that concurrently and comparably measures contextual (hippocampal) and stimulus-response (SR; striatal) memory by assessing their respective influences on attention in visual search (Goldfarb et al. 2016). We hypothesized that the least- and most-stressed participants from the population would differ in their ability to encode and retrieve contextual and SR associations. Specifically, if the relationship between lifetime stress and context memory followed the same trajectory as acute stress and hippocampal memory, then we would expect that very low stress participants would show impaired context memory. Thus, if only one memory system could be used, low stress participants would preferentially engage SR memory. On the other hand, if the relationship between lifetime stress and stimulus-response memory followed the same pattern as lifetime stress and addiction risk, we would expect that very low stress would be associated with impaired SR memory. In this case, we would expect low stress participants to preferentially engage context memory.

\section{Materials and Methods}

\section{Participants}

Participants $(N=893)$ completed the Stress and Adversity Inventory for Adults (STRAIN) online for course credit. This population consisted of healthy, young (in the final laboratory sample, $M=19.7 \mathrm{yr}, \mathrm{SD}=1.2$ ) participants who were completing full-time coursework at the time of assessment. Based on previous power analyses (Goldfarb et al. 2016), we invited STRAIN-screened participants into the laboratory until 35 participants from the bottom $25 \%$ and 35 participants from the top $25 \%$ of the distribution completed the laboratory study (Supplemental Table S1; see STRAIN below for details). Two additional laboratory participants were excluded due to failure to follow task instructions $(N=1)$ and incorrect coding of STRAIN responses $(N=1)$. Participants in the low- and moderate-stress groups did not differ with respect to age $(P>0.25)$ or gender $(P=0.19)$ (Supplemental Table $S 1)$. The laboratory session was completed for monetary compensa- tion within a few months of the STRAIN $(M=79 \mathrm{~d})$. Participants provided written informed consent for both components. All procedures were approved by the New York University Committee on Activities Involving Human Subjects.

\section{Materials}

\section{STRAIN}

Lifetime stress exposure was assessed using the Stress and Adversity Inventory for Adults (Adult STRAIN). The STRAIN employs a sophisticated interviewing methodology that includes extensive branching logic to assess a person's lifetime experience of 96 different acute and chronic stressors. If a respondent indicates that he or she has experienced a particular stressor, the branching logic prompts follow-up questions to assess the frequency, timing, duration, and perceived severity of the reported stressor. As such, the STRAIN provides extensive information on stressor exposure, the stressfulness of that exposure, when it occurred, and how long it lasted. The two main indices used in the present analyses were participants' lifetime stressor count and overall stressor severity, calculated as the sum of perceived severities of all reported stressors. Early life stress exposure was calculated by summing stressor counts and severity scores for all stressors occurring before age 18 , and recent stressors were defined as those that were either ongoing or experienced in the 3 mo prior to assessment. The STRAIN has good predictive validity, as evidenced by prior research showing that the main stress exposure indices from the STRAIN strongly predict several different health outcomes (Bower et al. 2014; Toussaint et al. 2016; Dooley et al. 2017). The Adult STRAIN also has high reliability, with a test-retest correlation of $r=0.92$ over $2 \mathrm{wk}$.

In order to compare individuals with low lifetime stress to those with moderate stress exposure, we computed the overall stressor severity score for each participant $(M=30.9$, range $=0$ 124) and recruited participants from the top and bottom $25 \%$ of this distribution to participate in the search task. Using a latent class analysis (Supplemental Fig. S1; Supplemental Text), we determined that the distribution of severity scores was better described by two latent classes than by one class, and that participants from the top and bottom of the distribution fell into distinct classes. These findings provide statistical support for dichotomizing the stress severity score and treating the participants from the top and bottom of the distribution as separate groups (MacCallum et al. 2002).

Participants from the bottom of the distribution $(N=35$ from the bottom $25 \%$ of our population) had experienced fewer than 4 major stressors on average (range $=0-11$ ), which places them at the extreme low-end of a larger population. However, participants reporting the highest stress severity $(N=35$ from the top $25 \%$ ) experienced an average of 29 lifetime stressors (range $=18-$ 50), which equates to a moderate level of lifetime stress exposure on the STRAIN (high stress participants, similar age group: $M=42$ stressors, range $=25-83$; G. M. Slavich and G. S. Shields, unpublished data), consistent with previous reports of relatively low stress exposure in this population (Anders et al. 2012). We thus refer to these groups as Low Stress (LS) and Moderate Stress (MS), respectively.

\section{Multicued search task}

To measure hippocampal and striatal memory we used a recently developed visual search task (Fig. 1). The multicued search task builds on the contextual cueing task, in which repeated spatial contexts facilitate search performance. This "contextual cueing" effect has been extensively replicated (e.g., Chun and Jiang 1998, 2003; Greene et al. 2007; Manelis and Reder 2012; Giesbrecht et al. 2013) and shown to critically involve the hippocampus (Chun and Phelps 1999). The multicued search task also includes probabilistic stimulus-response (SR) associations, designed to measure striatal memory. Previous research has demonstrated that, in a group of participants not screened for stress exposure, the multicued task can lead to performance benefits 


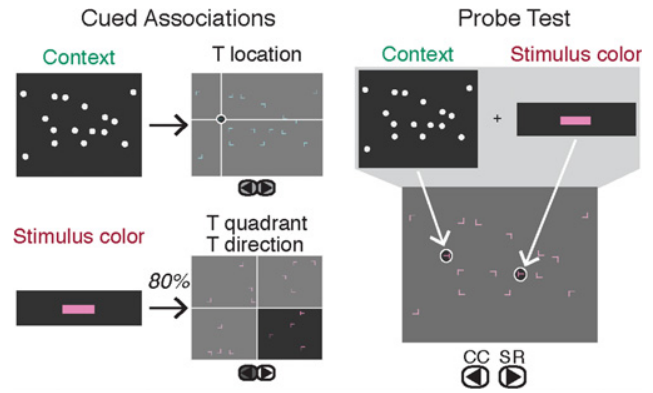

Figure 1. Search task to measure multiple memory systems. During the search phase (left), participants implicitly learned associations with mnemonic cues that could help them respond to the target. On contextually cued (CC) trials, repeated spatial patterns provided a cue for the location of the target. On stimulus-response (SR) trials, the color probabilistically cued the approximate target location and motor response. During the probe test (right), the two mnemonic cues were combined, each predicting a distinct target location and motor response.

from both types of memory cues, and that contextual and SR memory benefits are associated with hippocampal and striatal BOLD signal, respectively (Goldfarb et al. 2016). In addition to having been validated to involve these distinct memory systems, this task also provided several advantages for investigating the effects of stress on memory. First, the task enabled us to concurrently and comparably measure multiple memory systems. Second, the task limited the requirement for additional cognitive processes known to be affected by stress. For example, memory was measured without requiring participants to engage a particular strategy (as in Schwabe et al. 2007, 2008; Schwabe and Wolf 2012), thus avoiding the confound that stress influences strategy selection (Otto et al. 2013) and may differently impact the prefrontal circuitry (Arnsten 2009) involved in implementing hippocampal and striatal strategies (Dahmani and Bohbot 2015). Finally, the task includes search trials with no memory cues, but otherwise equivalent sensory and motor demands. By assessing use of memory relative to this individualized baseline, we can account for global stress-induced differences in task performance (Mather and Sutherland 2011).

The design of the task is explained in detail elsewhere (Goldfarb et al. 2016). Briefly, participants searched through visual displays for a target (a rotated " $\mathrm{T}$ ") among distractors. Participants had a maximum of 5 sec on each trial to locate the " $\mathrm{T}$ " and press a button indicating whether the " $\mathrm{T}$ " was rotated right or left. As soon as they responded, the screen disappeared and they were presented with feedback $(1 \mathrm{sec})$ on their performance (1-10 points for accurate responses, based on reaction time; 0 for missed responses, -10 for inaccurate responses). To measure hippocampal and striatal memory, a subset of trials contained mnemonic cues (Fig. 1, left). Contextually cued trials (CC, repeated $1 \times$ per block) consisted of displays with the same layout of shapes; thus, participants could use memory for the context to find the " $\mathrm{T}$ " (although this did not determine which button to press). On stimulus-response trials (SR) the shapes were presented in a new color (color repeated $5 \times$ per block). This color provided a cue (with $80 \%$ validity, or four out of five trials) to the quadrant in which the " $\mathrm{T}$ " appeared and the direction that the " $\mathrm{T}$ " was rotated; thus, participants could use memory for the probabilistic color-response association to find the " $\mathrm{T}$ " and press the correct button. These trials were randomly interleaved with trials with no memory cue (No Cue). The experiment had a yoked design: each pair of Low Stress/Moderate Stress participants completed the same experiment (i.e., same trial sequence, CC layouts, and SR color).

Participants completed the search task on two consecutive days (576 trials on Day 1, 192 on Day 2). On Day 2, they then completed explicit memory and probe tests. During the explicit memory test (Day 2, 48 trials), participants were told that they would see displays that they had viewed previously, but with the " $\mathrm{T}$ " re- moved. They were asked to indicate the quadrant in which the " $\mathrm{T}$ " had appeared (testing memory for CC and SR associations), and the direction of the "T" (SR). Finally, they completed a probe test (40 trials, Fig. 1, right). During this phase, participants were told that multiple "Ts" would appear on each trial, and to respond to the "T" they found first. Some trials included both CC and SR cues, each of which indicated the " $\mathrm{T}$ " would appear in a distinct quadrant (competitive, 12 trials) or in the same quadrant (collaborative, 4 trials). Additional trials with no memory cues (12 with "Ts" in distinct quadrants, 12 with "Ts" in the same quadrant) were randomly interleaved. As in the search phase, participants received feedback regarding reaction time (RT) and accuracy on every trial. Finally, participants were asked to verbally report any learned associations.

\section{Results}

\section{Influence of lifetime stress exposure on relative expression of multiple memory systems}

We first examined whether lifetime stress exposure, like acute stress, influenced which form of memory would be preferentially expressed. To do this, we analyzed performance during probe test trials in which participants could use either CC or SR memory (Fig. 1, right), focusing on the first trial in order to limit the influence of new learning. Lifetime stress exposure led to distinct memory biases-specifically, the majority of LS participants (71.4\%) used CC rather than SR memory, whereas the majority of MS participants (68.6\%) used SR rather than CC memory $\left(\chi_{(N=70)}^{2}=9.69, \quad P=0.004\right) \quad$ (Fig. 2). This pattern persisted throughout the probe test, with most LS (62.8\%) expressing CC memory and most MS (54.3\%) expressing SR memory. To understand how these memory biases developed, we examined how participants learned CC and SR associations.

\section{Influence of lifetime stress exposure on memory-guided search}

\section{Day 1}

Overall, visual search performance did not differ between the lowand moderate-stress groups (Accuracy-MS: $M=96.8 \%$, LS: $M=$ $96.1 \%, t_{(68)}=1.06, P>0.25$; RT-MS: $M=1.47$ sec, LS: $M=1.38$ sec, $t_{(68)}=1.23, P=0.22$; Fig. 3A). Participants in these two groups also did not differ in performance on No Cue trials

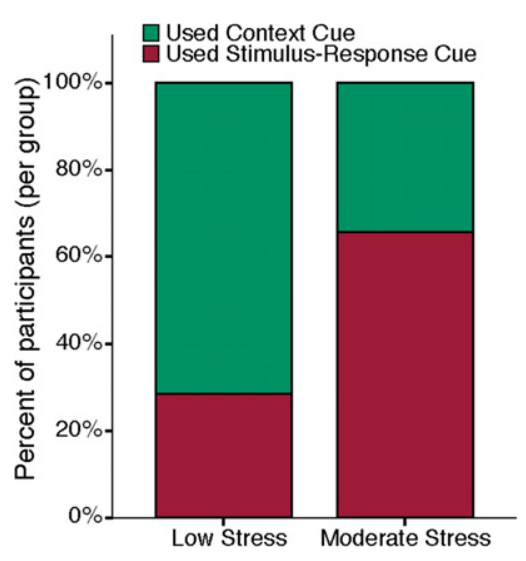

Figure 2. Proportion of participants choosing context or stimulus-response cued target during the first trial of the probe test. More Moderate Stress participants chose the target cued by the stimulus-response association, whereas more Low Stress participants chose the target cued by the context association. 
A

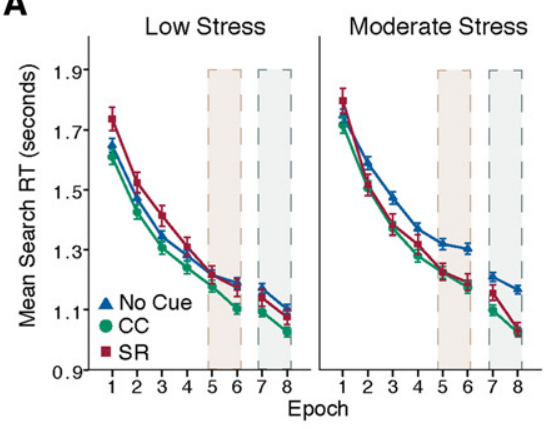

B

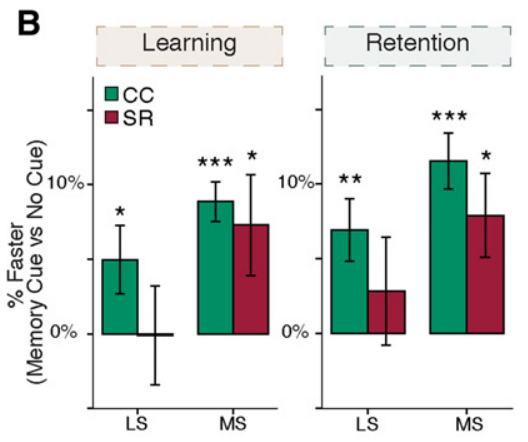

C

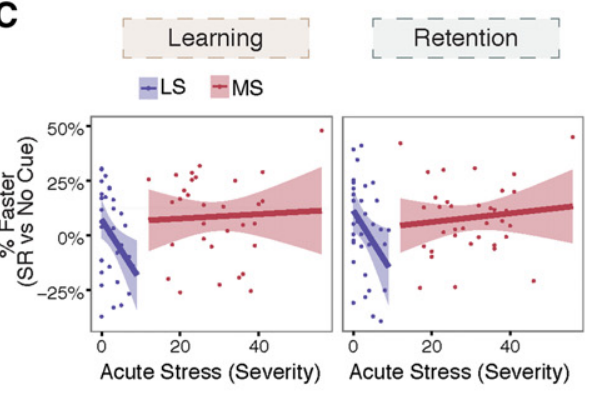

Figure 3. Search task performance. (A) Learning during the search task on Day 1 (Epoch 1-6) and retention on Day 2 (Epoch 7-8) for Low Stress (left) and Moderate Stress (right) participants. (B) Memory-guided search during the end of Day 1 (left) and Day 2 (right). Positive values indicate faster reaction time (RT) when using a memory cue compared with no memory cue. $\left({ }^{* * *}\right) P<0.001 ;\left(^{* *}\right) P<0.01 ;(*) P<0.05$. (C) STRAIN variables predict SR performance during learning (left) and retention (right). Severity of acute stressors negatively correlated with SR performance in Low Stress participants during learning and retention. Full list of predictive STRAIN variables in Supplemental Table S2. (CC) Contextually cued, (SR) stimulus-response cue, (LS) Low Stress, (MS) Moderate Stress.

(Accuracy- $t_{(68)}=1.12, P>0.25 ;$ RT-Main Effect of Group: $F_{(1,68)}=2.5, P=0.12, \eta_{p}^{2}=0.035$; Group $\times$ Epoch: $F_{(1,68)}=1.19$, $\left.P=0.28, \eta_{p}^{2}=0.017\right)$. Further, there were no differences in accuracy between trial types, demonstrating that participants could perform the task with and without memory cues, and that differences in RT are not due to speed/accuracy tradeoffs (Supplemental Table S2). As in previous studies (Chun and Jiang 1998; Goldfarb et al. 2016), we measured the influence of memory on search as performance on trials with memory cues relative to trials with no mnemonic cue (No Cue trials) during the second half of the experiment. To enable comparison with Day 2 performance, we focus on the last two epochs of Day 1.

Lifetime stress exposure led to differences in learning and retrieval of SR associations. Although MS participants showed significant SR learning at the end of Day $1\left(t_{(34)}=2.12, P=0.041\right)$, LS participants did not $\left(t_{(34)}=-0.83, P>0.25\right)$. These patterns were consistent throughout Day 1 (MS: Cue [No Cue vs. SR] $\times$ Time: $\quad F_{(5,170)}=2.58, \quad P=0.042$, Huynh-Feldt corrected, $\eta_{p}^{2}=0.071$; LS: Cue $\times$ Time: $\left.F_{(5,170)}=1.45, P>0.21\right)$. As SR cues were $80 \%$ valid, we could also assess development of SR associations by comparing performance on valid to invalid trials. We observed a significant Group $\times$ Cue [SR-Valid vs. SR-Invalid] interaction throughout Day $1\left(F_{(1,68)}=4.21, P=0.044, \eta_{p}^{2}=0.058\right)$, showing that SR memory differed between MS and LS participants (Supplemental Fig. S2).

In contrast, the stress groups did not differ in learning and retrieval of CC associations. Both groups showed significant CC learning during the last two epochs (faster RT on CC than No Cue trials; LS: $t_{(34)}=2.25, P=0.031$; MS: $\left.t_{(34)}=6.71, P<0.001\right)$. MS participants showed significant CC learning throughout Day 1 (Main effect of Cue [No Cue vs. CC]: $F_{(1,34)}=20.36, P<0.001$, $\eta_{p}^{2}=0.37$ ), while LS showed the same pattern (although not significant; $\left.F_{(1,34)}=2.56, P=0.12, \eta_{p}^{2}=0.07\right)$, with no significant difference between stress groups (Group $\times$ Cue: $F_{(1,68)}=1.3, P>$ $0.25)$.

\section{Day 2}

The above results persisted $24 \mathrm{~h}$ later (Fig. 3). Again, there was no significant difference between stress groups in overall search performance (Accuracy-MS: $M=97.9 \%$, LS: $M=97 \% ; t_{(68)}=1.51$, $P=0.14$; RT—MS: $1.13 \mathrm{sec}$; LS: $\left.1.11 \mathrm{sec} ; t_{(68)}=0.49, P>0.25\right)$ or performance on No Cue trials (Accuracy- $t_{(68)}=1.01, P>0.25$; RT $\left.-F_{(1,68)}=1.16, P>0.25, \eta_{p}^{2}=0.017\right)$. Although MS partici- pants continued to show SR memory expression (faster on SR than No Cue; $\left.t_{(34)}=2.59, P=0.014\right)$, LS participants still did not $\left(t_{(34)}=0.13, P>0.25\right)$. Both groups retained CC memory on Day 2 (faster on CC than No Cue; LS: $t_{(34)}=3.14, P=0.003$; MS: $\left.t_{(34)}=5.58, P<0.001\right)$.

In the explicit memory tests immediately following search, neither stress group showed above chance (25\%) memory for the cued "T" location on CC trials (LS: $M=27.5 \%, t_{(34)}=0.89$, $P>0.25$; MS: $\left.27.9 \%, t_{(34)}=1.07, P>0.25\right)$, or the cued "T" orientation on SR trials (50\% chance; LS: $52.9 \%, t_{(34)}=0.7, P>0.25$; MS: $\left.54.3 \%, t_{(34)}=0.9, P>0.25\right)$. However, MS participants were significantly above chance in recalling the cued " $\mathrm{T}$ " location on SR trials $\left(37.9 \%, t_{(34)}=3.43, P=0.002\right)$, whereas LS were not $\left(25.7 \%, t_{(34)}=0.21, P>0.25\right)$. Despite this, only one MS participant $(2.9 \%)$ verbally reported that the " $\mathrm{T}$ " usually appeared in a consistent location.

\section{Relationship between types of stress exposure and SR memory}

To examine whether specific aspects of lifetime stress exposure explained differences in SR learning and retention (beyond those explained by MS versus LS group), we conducted linear regressions in which Group and different STRAIN variables were used as predictors. SR learning and retention were quantified as the percent difference in RT between accurate No Cue and SR trials during the end of Day 1 and throughout Day 2, respectively. Based on prior research (Bower et al. 2014; Toussaint et al. 2016), we examined the frequency and perceived severity of acute, chronic, early life, and adulthood stressors as predictors of SR learning and retention in separate regressions (Supplemental Table S3).

For both learning and retention, we observed a main effect of acute stressor count (Day 1: $\beta=-4.8, P=0.017$; Day 2: $\beta=-5.81, P=0.003$ ), acute stressor severity (Day $1: \beta=-2.8$, $P=0.021$; Day 2: $\beta=-2.89, P=0.015$ ) (Fig. $3 \mathrm{C}$ ), and early life stressor count (Day 1: $\beta=-3.92, P=0.048$; Day 2: $\beta=-5.8$, $P=0.003)$, such that increasing stress was associated with worse SR performance. This relationship was driven by LS participants: for all stress indices listed above, increasing levels of stress negatively predicted SR performance in LS (all Ps $<0.05$ ) but not MS participants (all Ps $>0.11$ ). In other words, among those participants with very low overall stress exposure, those who had experienced more (or more severe) stressors showed worse SR performance. 


\section{Computational model: Understanding differences in SR memory}

Thus far, we have demonstrated that individuals who experienced moderate levels of lifetime stress exposure show faster RT in the presence of SR cues relative to trials with no memory cues, but those who experienced low levels of lifetime stress exposure do not show this RT benefit. We also showed that low stress participants do not differ in RT based on whether the SR cue is valid or invalid, although moderate stress participants show this distinction. It is possible that the stress groups differed in another dimension of task performance that led to this result. Further, if the groups did specifically differ in SR memory, it remains unclear what component of SR memory was altered in low stress participants.

To address these questions, we created a computational model to characterize dynamics influencing RT on each trial of Day 1. This allowed us to examine the contribution of the different memory systems in terms of trial-by-trial RT adjustments reflecting learning, complementary to the epoch-wise analyses presented above. We modeled RT on every trial as reflecting a nonspecific improvement with total time on task, accompanied by trialspecific contributions of three learning processes: priming of stimulus locations (regardless of trial cue type), and learning based on history with CC and SR cues.

As reported previously (Maljkovic and Nakayama 1996), RT provided evidence of location priming. Participants were significantly faster to find the " $\mathrm{T}$ " if it was in the same quadrant $(M=$ $1.33 \mathrm{sec})$ rather than a different quadrant $(1.45 \mathrm{sec})$ than the previous trial $\left(t_{(69)}=7.63, P<0.001\right)$. A similar process occurred on SR trials: participants were significantly faster to find the " $\mathrm{T}$ " when it was in the same quadrant (SR-Valid; $1.39 \mathrm{sec}$ ) rather than a different quadrant (SR-Invalid; $1.44 \mathrm{sec}$ ) than the last SR trial $\left(t_{(69)}=2.24, P=0.028\right)$. Thus, RT could reflect memory for the " $\mathrm{T}$ " location on the previous trial and, on CC or SR trials, memory for the " $\mathrm{T}$ " location on the previous trial with that cue.

Specifically, for every trial $t$, we modeled RT as follows:

$$
\begin{aligned}
\log \left(\mathrm{RT}_{t}\right)= & \beta_{0}+\left(\beta_{\mathrm{tNum}} \times \log \left(\mathrm{tNum}_{t}\right)\right)+\left(\beta_{\text {prime }} \times w_{q}^{\text {prime }}\right) \\
& +\mathrm{ISR}_{t} \times\left(\beta_{\mathrm{SR}} \times w_{q}^{\mathrm{SR}}\right)+\mathrm{ICC}_{t} \times\left(\beta_{\mathrm{CC}} \times w_{q}^{\mathrm{CCn}}\right)+\varepsilon_{t}
\end{aligned}
$$

where $\mathrm{ISR}_{t}$ and $\mathrm{ICC}_{t}$ are binary indicators of whether trial $t$ was a CC or SR trial and $q$ indicates the correct quadrant.

The vectors $\vec{w}$ updated on every relevant trial following a prediction-error based Rescorla-Wagner updating rule (Rescorla and Wagner 1972), with a stepsize determined by a correspond- ing learning rate $\left(\alpha_{\text {prime, }} \alpha_{\mathrm{SR}}\right.$, or $\alpha_{\mathrm{CC}}$; details in Supplemental Text).

The $\alpha$ 's and $\beta$ 's, all free parameters that we fit to best explain each subject's RT timeseries, govern the acquisition and expression (respectively) of the learned associations that guide search. Accordingly, this model embodies two distinct (but not mutually exclusive) hypotheses that could explain the difference in performance between MS and LS participants.

One possibility is MS participants were better at using the $\vec{w}^{\mathrm{SR}}$ predictions to facilitate RT on SR-cued trials, which would be shown by a significantly lower $\beta_{\mathrm{SR}}$. Another possibility is that MS participants were faster to update location expectations on SR-cued trials, which would be reflected by a significantly larger learning rate $\alpha_{\mathrm{SR}}$. We used Bayesian hierarchical modeling (Supplemental Text) to derive group-level estimates of each parameter and thereby to estimate the difference in each parameter between groups. If the change in parameter estimates from MS to LS participants was significantly different from zero, the two groups differed on that parameter.

The model captured the learning trends seen in the data (Fig. 4A). The only parameter that differed significantly between MS and LS participants was $\beta_{\mathrm{SR}}$, showing that expression of SR learning was reduced in LS participants (Fig. 4B; full parameter list in Supplemental Table S4). That is, although the MS group used location expectations formed on previous SR trials to find the "T" more quickly, the LS group made significantly less use of these SR expectations. However, we did not find evidence that the updating of location expectations differed between groups, as there was no significant difference in learning rate $\left(\alpha_{S R}\right)$. These results indicate that lifetime stress exposure modulated the use, while not significantly changing the learning rate, of stimulus-response associations.

\section{Discussion}

The present data provide some of the first evidence that very low levels of lifetime stress exposure can have negative effects on memory. Although individuals with low levels of lifetime stress did not differ from moderately stressed participants in overall visual search performance or in learning and memory for contextual associations, they did differ in stimulus-response (SR) memory. Individuals who had experienced low levels of lifetime stress made significantly less use of probabilistic stimulus-response associations than those who had experienced moderate levels of stress. These effects were supported by a computational model of task performance, showing that the only parameter that significantly differed between groups was the parameter governing the expression of SR memory.
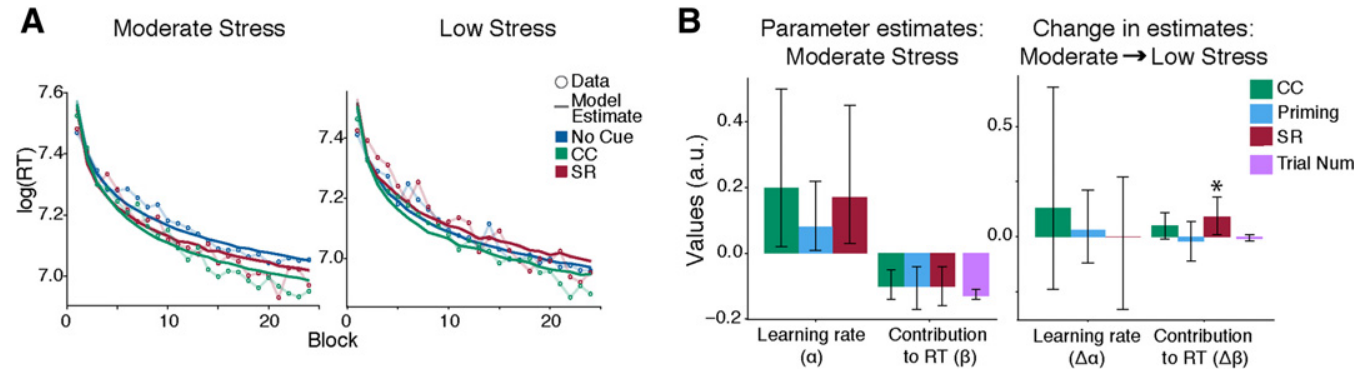

Figure 4. Computational model of visual search performance. (A) Model estimates (lines) and actual reaction time data (open circles) are shown for Moderate Stress (left) and Low Stress (right) participants, per block of search during Day 1. (B) Derived estimates of model parameter values for Moderate Stress (left) participants are shown, along with any change in these parameters that was necessary to describe Low Stress participants (right). Error bars represent $95 \%$ confidence intervals. When the range indicated by these bars does not include zero, this estimate is significantly different from zero. The only change parameter that differed from zero was $\beta_{S R}$. All parameters described in Supplemental Text. 
The results of the probe test, demonstrating that higher levels of stress are associated with increased likelihood of using SR rather than context memory, are consistent with a large body of research. In both rodents and humans, studies have consistently shown that recent chronic (rodent: Schwabe et al. 2008; Ferragud et al. 2010; human: Schwabe et al. 2008) and acute stress (rodent: Kim et al. 2001; Packard and Wingard 2004; Packard 2009; Schwabe et al. 2010; Vanelzakker et al. 2011; Leong and Packard 2014; human: Schwabe et al. 2007; 2013; Schwabe and Wolf 2012; but see Schwabe et al. 2009) causes a shift from expressing hippocampal to striatal memory. However, as only one memory system could be expressed in these tasks, and these systems are known to compete (Poldrack and Packard 2003; Foerde et al. 2006) or compensate (Moody et al. 2004), it was not clear how each form of memory was affected individually (Goldfarb and Phelps 2017). Here, we show that the trade-off between context and SR memory with varying lifetime stress was driven by differences in SR memory.

These results demonstrate that context and SR memory are differentially sensitive to very low levels of lifetime stress. Although expression of SR memory differed between low and moderate stress participants, context memory did not. Our results do not support the hypothesis that very low lifetime stress would, like low levels of acute arousal (McCullough et al. 2015), lead to impaired hippocampal memory in the low stress group. Instead, we found support for our hypothesis that SR memory would be reduced among low stress participants. This result is consistent with prior research showing that higher cumulative stress is associated with increasing odds of alcohol dependence (Lloyd and Turner 2008), which has itself been theorized to result from enhanced striatal memory (McDonald et al. 2004). Thus, it is possible that mechanisms shown to link lifetime stress to addiction risk in preclinical populations (see Sinha 2008 for review) are also relevant to changes in striatal memory.

Despite this group-level effect, we were surprised to find a negative relationship between stressors and expression of SR memory among participants with overall low stress exposure. This may be due in part to the fact that individual stressors occur within the context of a lifetime of events (Seery et al. 2010) and attain greater importance among individuals with overall very few stressful events. One possibility is that maladaptive responses to stressors among those with low stress exposure (Seery et al. 2013; Liu 2015) could contribute to performance deficits. For example, recent work has shown lower striatal BOLD signal in response to stress among individuals with low cumulative adversity (Seo et al. 2014). Given the previously reported relationship between increased striatal signal and SR memory in the present task (Goldfarb et al. 2016), generally dampened striatal reactivity could lead to worse performance. Although future studies are needed to understand the mechanism by which this negative relationship occurs, this finding emphasizes the importance of treating cumulative stress as a continuous as well as dichotomized variable.

Limitations of the present study include simultaneous measurement of contextual and stimulus-response memory. Given potential interactions and competition between these memory systems (Poldrack and Packard 2003; McDonald et al. 2004; Foerde et al. 2006), it is unclear whether the observed reduction in SR expression among LS participants would exist in a task that assessed only SR learning. However, there are rarely cases in everyday life that engage only one memory system. Even in canonical SR tasks, the medial temporal lobe has been shown to contribute to learning (Poldrack et al. 2001), lending the current approach greater ecological validity. Another limitation involves the use of retrospective self-report to assess lifetime stressors. Because we measured (but did not manipulate) lifetime stress ex- posure, these results do not demonstrate a causal relationship between stress and memory. The validity of using self-report to assess stress exposure has been demonstrated in numerous studies: many life events can be reliably recalled when probed systematically (Brewin et al. 1993), and self-reported stress exposure has been associated with different neural (Seo et al. 2014) and behavioral responses to acute stressors (Seery et al. 2013), as well as different health outcomes (Bower et al. 2014; Toussaint et al. 2016). It is also unlikely that observed differences in learning are the result of systematic biases in self-reported stress exposure. Nonetheless, future research should examine the present associations using independent measures of objective stress exposure. Finally, these data do not address the effects of extremely high levels of cumulative stress exposure. The young undergraduate population used in this study had experienced relatively moderate levels of stress exposure relative to the general population, which is an appropriate sample in which to test the present hypotheses. An additional "high stress" community sample could be added in future research, but caution is warranted, as such a sample would differ in many demographic characteristics and thus potentially confound comparisons between groups. Nevertheless, additional research is needed to understand the effects of the full trajectory of lifetime stress exposure on the use of different memory systems.

In sum, the present study provides preliminary evidence that very low levels of lifetime stress exposure may be associated with impairments in cognitive functions in humans. Relative to participants who had experienced moderate levels of lifetime stress, low stress participants showed reduced expression of stimulus-response memory. This difference was specific to stimulus-response memory, as low stress participants were capable of learning and retrieving context memories. These results raise the question of whether some stress exposure is necessary for good stimulus-response memory, and how very low levels of stress exposure could influence other cognitive processes.

\section{Acknowledgments}

The authors are grateful to Marvin Chun for helpful discussions. This research was supported by a National Science Foundation Graduate Research Fellowship (E.V.G.), NIH grant K08 MH103443 (G.M.S.), a Society in Science-Branco Weiss Fellowship (G.M.S.), and NIH grant 1R01MH097085 (E.A.P.).

\section{References}

Anders SL, Frazier PA, Shallcross SL. 2012. Prevalence and effects of life event exposure among undergraduate and community college students. I Couns Psychol 59: 449-457.

Arnsten AF. 2009. Stress signalling pathways that impair prefrontal cortex structure and function. Nat Rev Neurosci 10: $410-422$.

Bayley PJ, Frascino JC, Squire LR. 2005. Robust habit learning in the absence of awareness and independent of the medial temporal lobe. Nature 436: 550-553.

Bower JE, Crosswell AD, Slavich GM. 2014. Childhood adversity and cumulative life stress: risk factors for cancer-related fatigue. Clin Psychol Sci 2: 108-115.

Breslau N, Davis GC, Andreski P, Peterson E. 1991. Traumatic events and posttraumatic stress disorder in an urban population of young adults. Arch Gen Psychiatry 48: 216-222.

Brewin CR, Andrews B, Gotlib IH. 1993. Psychopathology and early experience: a reappraisal of retrospective reports. Psychol Bull 113: 82-98.

Bunsey M, Eichenbaum H. 1996. Conservation of hippocampal memory function in rats and humans. Nature 379: 255-257.

Burgess N, Maguire EA, O'Keefe J. 2002. The human hippocampus and spatial and episodic memory. Neuron 35: 625-641.

Chun MM, Jiang Y. 1998. Contextual cueing: implicit learning and memory of visual context guides spatial attention. Cogn Psychol 36: 28-71.

Chun MM, Jiang Y. 2003. Implicit, long-term spatial contextual memory. $J$ Exp Psychol Learn Mem Cogn 29: 224-234. 
Chun MM, Phelps EA. 1999. Memory deficits for implicit contextual information in amnesic subjects with hippocampal damage. Nat Neurosci 2: 844-847.

Dahmani L, Bohbot VD. 2015. Dissociable contributions of the prefrontal cortex to hippocampus- and caudate nucleus-dependent virtual navigation strategies. Neurobiol Learn Mem 117: 42-50.

Dias-Ferreira E, Sousa JC, Melo I, Morgado P, Mesquita AR, Cerqueira JJ, Costa RM, Sousa N. 2009. Chronic stress causes frontostriatal reorganization and affects decision-making. Science 325: 621-625.

Dienstbier RA. 1989. Arousal and physiological toughness: implications for mental and physical health. Psychol Rev 96: 84-100.

Dooley LN, Slavich GM, Moreno PI, Bower JE. 2017. Strength through adversity: moderate lifetime stress exposure is associated with psychological resilience in breast cancer survivors. Stress Health. doi: $10.1002 /$ smi.2739.

Elliott AE, Packard MG. 2008. Intra-amygdala anxiogenic drug infusion prior to retrieval biases rats towards the use of habit memory. Neurobiol Learn Mem 90: 616-623.

Elzinga BM, Roelofs K, Tollenaar MS, Bakvis P, van Pelt J, Spinhoven P. 2008. Diminished cortisol responses to psychosocial stress associated with lifetime adverse events a study among healthy young subjects. Psychoneuroendocrinology 33: 227-237.

Evans GW, Schamberg MA. 2009. Childhood poverty, chronic stress, and adult working memory. Proc Natl Acad Sci 106: 6545-6549.

Ferragud A, Haro A, Sylvain A, Velazquez-Sanchez C, Hernandez-Rabaza V, Canales JJ. 2010. Enhanced habit-based learning and decreased neurogenesis in the adult hippocampus in a murine model of chronic social stress. Behav Brain Res 210: 134-139.

Foerde K, Knowlton BJ, Poldrack RA. 2006. Modulation of competing memory systems by distraction. Proc Natl Acad Sci 103: 11778-11783.

Giesbrecht B, Sy JL, Guerin SA. 2013. Both memory and attention systems contribute to visual search for targets cued by implicitly learned context. Vision Res 85: 80-89.

Goldfarb EV, Phelps EA. 2017. Stress and the trade-off between hippocampal and striatal memory. Curr Opin Behav Sci 14: 47-53.

Goldfarb EV, Chun MM, Phelps EA. 2016. Memory-guided attention: independent contributions of the hippocampus and striatum. Neuron 89: $317-324$.

Goodman J, Packard MG. 2016. Memory systems and the addicted brain. Front Psychiatry 7: 24.

Graybiel AM. 1998. The basal ganglia and chunking of action repertoires. Neurobiol Learn Mem 70: 119-136.

Greene AJ, Gross WL, Elsinger CL, Rao SM. 2007. Hippocampal differentiation without recognition: an fMRI analysis of the contextual cueing task. Learn Mem 14: 548-553.

Kim JJ, Lee HJ, Han JS, Packard MG. 2001. Amygdala is critical for stress-induced modulation of hippocampal long-term potentiation and learning. J Neurosci 21: 5222-5228.

Leong KC, Packard MG. 2014. Exposure to predator odor influences the relative use of multiple memory systems: role of basolateral amygdala. Neurobiol Learn Mem 109: 56-61.

Liu RT. 2015. A developmentally informed perspective on the relation between stress and psychopathology: when the problem with stress is that there is not enough. J Abnorm Psychol 124: 80-92.

Lloyd DA, Turner RJ. 2008. Cumulative lifetime adversities and alcohol dependence in adolescence and young adulthood. Drug Alcohol Depend 93: $217-226$.

Lovallo WR, Farag NH, Sorocco KH, Cohoon AJ, Vincent AS. 2012. Lifetime adversity leads to blunted stress axis reactivity: studies from the Oklahoma Family Health Patterns Project. Biol Psychiatry 71: 344-349.

Lupien SJ, Maheu F, Tu M, Fiocco A, Schramek TE. 2007. The effects of stress and stress hormones on human cognition: implications for the field of brain and cognition. Brain Cogn 65: 209-237.

Lupien SJ, McEwen BS, Gunnar MR, Heim C. 2009. Effects of stress throughout the lifespan on the brain, behaviour and cognition. Nat Rev Neurosci 10: $434-445$.

MacCallum RC, Zhang S, Preacher KJ, Rucker DD. 2002. On the practice of dichotomization of quantitative variables. Psychol Methods 7: 19-40.

Maljkovic V, Nakayama K. 1996. Priming of pop-out: II. The role of position. Percept Psychophys 58: 977-991.

Manelis A, Reder LM. 2012. Procedural learning and associative memory mechanisms contribute to contextual cueing: evidence from fMRI and eye-tracking. Learn Mem 19: 527-534.

Mather M, Sutherland MR. 2011. Arousal-biased competition in perception and memory. Perspect Psychol Sci 6: 114-133.

McCullough AM, Ritchey M, Ranganath C, Yonelinas AP. 2015. Differential effects of stress-induced cortisol responses on recollection and familiarity-based recognition memory. Neurobiol Learn Mem 123: 1-10.

McDonald RJ, Devan BD, Hong NS. 2004. Multiple memory systems: the power of interactions. Neurobiol Learn Mem 82: 333-346.
McEwen BS. 1998. Stress, adaptation, and disease. Allostasis and allostatic load. Ann N Y Acad Sci 840: 33-44.

McEwen BS. 1999. Stress and hippocampal plasticity. Annu Rev Neurosci 22: $105-122$.

Moody TD, Bookheimer SY, Vanek Z, Knowlton BJ. 2004. An implicit learning task activates medial temporal lobe in patients with Parkinson's disease. Behav Neurosci 118: 438-442.

Otto AR, Raio CM, Chiang A, Phelps EA, Daw ND. 2013. Working-memory capacity protects model-based learning from stress. Proc Natl Acad Sci 110: $20941-20946$.

Packard MG. 2009. Anxiety, cognition, and habit: a multiple memory systems perspective. Brain Res 1293: 121-128.

Packard MG, Wingard JC. 2004. Amygdala and "emotional" modulation of the relative use of multiple memory systems. Neurobiol Learn Mem 82: $243-252$.

Patterson TK, Craske MG, Knowlton BJ. 2013. The effect of early-life stress on memory systems supporting instrumental behavior. Hippocampus 23: $1025-1034$

Poldrack RA, Packard MG. 2003. Competition among multiple memory systems: converging evidence from animal and human brain studies. Neuropsychologia 41: 245-251.

Poldrack RA, Clark J, Pare-Blagoev EJ, Shohamy D, Creso Moyano J, Myers C, Gluck MA. 2001. Interactive memory systems in the human brain. Nature 414: $546-550$.

Rescorla RA, Wagner AR. 1972. A theory of Pavlovian conditioning: variations in the effectiveness of reinforcement and nonreinforcement. In Classical conditioning II: current research and theory (ed. Black AH, Prokasy WF), pp. 64-99. Appleton-Century-Crofts, New York.

Schwabe L, Wolf OT. 2012. Stress modulates the engagement of multiple memory systems in classification learning. J Neurosci 32: 11042-11049.

Schwabe L, Oitzl MS, Philippsen C, Richter S, Bohringer A, Wippich W, Schachinger H. 2007. Stress modulates the use of spatial versus stimulus-response learning strategies in humans. Learn Mem 14: 109-116.

Schwabe L, Dalm S, Schachinger H, Oitzl MS. 2008. Chronic stress modulates the use of spatial and stimulus-response learning strategies in mice and man. Neurobiol Learn Mem 90: 495-503.

Schwabe L, Oitzl MS, Richter S, Schachinger H. 2009. Modulation of spatial and stimulus-response learning strategies by exogenous cortisol in healthy young women. Psychoneuroendocrinology 34: 358-366.

Schwabe L, Schachinger H, de Kloet ER, Oitzl MS. 2010. Corticosteroids operate as a switch between memory systems. J Cogn Neurosci 22: $1362-1372$.

Schwabe L, Bohbot VD, Wolf OT. 2012. Prenatal stress changes learning strategies in adulthood. Hippocampus 22: 2136-2143.

Schwabe L, Tegenthoff M, Hoffken O, Wolf OT. 2013. Mineralocorticoid receptor blockade prevents stress-induced modulation of multiple memory systems in the human brain. Biol Psychiatry 74: 801-808.

Seery MD, Holman EA, Silver RC. 2010. Whatever does not kill us: cumulative lifetime adversity, vulnerability, and resilience. J Pers Soc Psychol 99: 1025-1041.

Seery MD, Leo RJ, Lupien SP, Kondrak CL, Almonte JL. 2013. An upside to adversity?: moderate cumulative lifetime adversity is associated with resilient responses in the face of controlled stressors. Psychol Sci 24: 1181-1189.

Seo D, Tsou KA, Ansell EB, Potenza MN, Sinha R. 2014. Cumulative adversity sensitizes neural response to acute stress: association with health symptoms. Neuropsychopharmacology 39: 670-680.

Sinha R. 2008. Chronic stress, drug use, and vulnerability to addiction. Ann N Y Acad Sci 1141: 105-130.

Taylor SB, Anglin JM, Paode PR, Riggert AG, Olive MF, Conrad CD. 2014. Chronic stress may facilitate the recruitment of habit- and addiction-related neurocircuitries through neuronal restructuring of the striatum. Neuroscience 280: 231-242.

Toussaint L, Shields GS, Dorn G, Slavich GM. 2016. Effects of lifetime stress exposure on mental and physical health in young adulthood: how stress degrades and forgiveness protects health. J Health Psychol 21: 1004-1014.

Vanelzakker MB, Zoladz PR, Thompson VM, Park CR, Halonen JD, Spencer RL, Diamond DM. 2011. Influence of pre-training predator stress on the expression of c-fos mRNA in the hippocampus, amygdala, and striatum following long-term spatial memory retrieval. Front Behav Neurosci 5: 30.

Yin HH, Knowlton BJ. 2006. The role of the basal ganglia in habit formation. Nat Rev Neurosci 7: 464-476.

Received January 30, 2017; accepted in revised form February 21, 2017. 


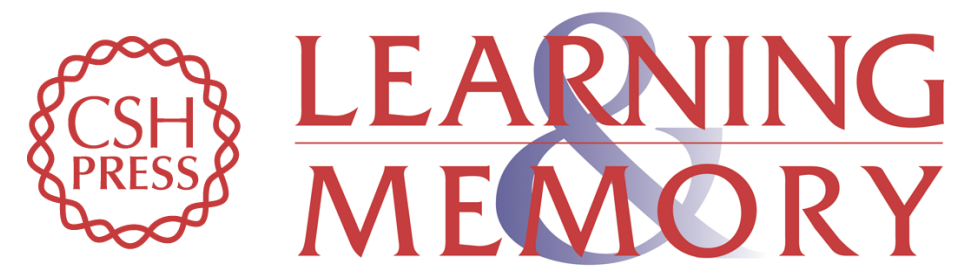

\title{
Low lifetime stress exposure is associated with reduced stimulus- response memory
}

\author{
Elizabeth V. Goldfarb, Grant S. Shields, Nathaniel D. Daw, et al.
}

Learn. Mem. 2017, 24:

Access the most recent version at doi:10.1101/lm.045179.117

\section{Supplemental http://learnmem.cshlp.org/content/suppl/2017/03/15/24.4.162.DC1 Material}

References This article cites 63 articles, 9 of which can be accessed free at: http://learnmem.cshlp.org/content/24/4/162.full.html\#ref-list-1

Creative This article is distributed exclusively by Cold Spring Harbor Laboratory Press for the Commons first 12 months after the full-issue publication date (see

License http://learnmem.cshlp.org/site/misc/terms.xhtml). After 12 months, it is available under a Creative Commons License (Attribution-NonCommercial 4.0 International), as described at http://creativecommons.org/licenses/by-nc/4.0/.

Email Alerting Receive free email alerts when new articles cite this article - sign up in the box at the Service top right corner of the article or click here. 